\title{
Algorithmic Analysis of an Efficient Packet Scheduler for Optimizing the QoS of VolP Networks
}

\author{
J. N. Dike \\ Department of Electronic/ \\ Computer Engineering \\ University of Port Harcourt \\ Port Harcourt, Nigeria
}

\author{
C. I. Ani \\ Department of Electronic \\ Engineering \\ University of Nigeria \\ Nsukka, Nigeria
}

\begin{abstract}
Owing to the emerging realities in the Information and Communication Technology (ICT) industry, where data traffic is growing much faster than traditional voice traffic, there is now a global growing desire to migrate to digital form of communication. The trend is focused in transporting voice over data networks rather than the traditional data over voice networks. Voice traffic carried over a system originally designed for data creates technical challenges. This is primarily due to limitations that have resulted from the nature of the Internet and its bandwidth, transmission impairments and voice compression technology, which degrade voice quality. In this paper, the design of a hybrid architecture of an efficient packet scheduler for optimizing the QoS of VoIP networks have been presented. The design addresses the transmission impairment factors of delay (or latency), jitter (or delay variation) and packet loss. Mechanisms for: accommodating the demands of the expected rapid increase in the volume of voice traffic as PSTN progressively migrates to VoIP; according due precedence to the delay-sensitive voice and business/mission critical data (B/MCD) traffic flows; ensuring fair resource sharing among all traffic flows (realtime and non real-time) and adaptively maintaining optimal voice quality without over provisioning the users, have been incorporated. In the analysis, the algorithm defining the different levels of services was developed and explained. The interaction and integration of the designed functional pieces were used to develop a structured signal flowchart. The work therefore provides a theoretical framework that guarantees a graceful tradeoff between priority and fairness to all traffics flows running on the network.
\end{abstract}

\section{General Terms}

Hybrid Architecture, Algorithmic Analysis, Quality of Service, Voice over Internet Protocol Network, Transmission Impairment Factor, Structured Design.

\section{Keywords}

Business/Mission Critical Data, Packet Scheduler, Signal Flowchart, Converged Network, Packet Classifier, Token Bucket, Modular, Abstraction, Optimization.

\section{INTRODUCTION}

The advent of packet-switched networks has brought with it more efficient and affordable means of data transfer across the globe. Consequently, these networks are currently evolving into a converged, integrated (voice/data/video), Internet Protocol (IP)-based network layer that runs over a highcapacity transport infrastructure. The interesting reality is that this converged network promises to deliver lower operating costs and easier service deployment [1]. A good example of a converged network is Voice over Internet Protocol (VoIP) network. VoIP entails transmitting voice traffic across a packet-switched (IP) network, for example, the Internet. It promises to integrate the data and voice networks into a single network. VoIP is therefore one of the most important technologies in the world of communications today and is the most important service in the broadband network with growing attempts focused on improving voice quality to match that in the PSTN $[2,3]$. Convergence has become a vital tool for providing the balance of cost reduction and providing increased capacity for improved services.

Increasingly, the expectation placed upon VoIP networks is that they will provide the same or better voice quality than traditional telephone, thereby assuring Quality of Service (QoS) for voice traffic over the network. QoS implies the ability of a network to satisfy voice traffic and service requirements [4]. That is, the ability of a network to achieve maximum bandwidth and deal with other network performance factors such as latency, jitter, error rate and uptime. It also entails controlling and managing network resources by setting priorities for specific types of traffic on the network. The migration of telephone services from PSTN to Internet telephony (or VoIP) has been on the increase within the last two decades [5, 6, 7]. Since Internet access has become a global possibility, this reported trend of migration of telephone services to VoIP is the same all over the globe.

But if one expects to run long-distance phone calls over a network, he/she should determine ahead of time if the network will provide good voice quality for the remote locations. Although developing rapidly, Internet telephony still has some problems with reliability and sound quality. The topology and behavior of circuit-switched networks and data networks are significantly different. Voice traffic carried over a system originally designed for data creates technical challenges. This is primarily due to limitations that resulted from the nature of the Internet and its bandwidth, transmission impairments and compression technology, which degrade voice quality $[8,9$, $10,11,12]$. The original Internet architecture was designed for best effort traffics and therefore does not guarantee QoS for real-time applications.

The expected rapid increase of voice traffic in the Internet, demands the design of robust packet monitoring and scheduling architectures. Again, there isn't much research done in offering special preference to business/mission critical data $(\mathrm{B} / \mathrm{MCD})$ traffic (such as real-time online purchases, security alerts, bank transfers, weather forecasts, remote/emergency environmental monitoring, disaster alerts, military commands, remote industrial control systems, and so 
on) as accorded to voice traffic. In other words, earlier solutions to the QoS challenges of VoIP networks have been focused on giving precedence to voice traffic at the expense of $\mathrm{B} / \mathrm{MCD}$ traffics. To ensure good voice quality in a data network these challenges must be addressed if VoIP will serve as the ultimate alternative for telephone applications. Over the years, the telecommunication industry and research interests have been focused on possible ways of combating the inherent transmission impairments. The full deployment of VoIP technology therefore currently awaits the development and deployment of appropriate schemes to address these QoS assessment parameters.

Standing upon these observations and conviction therefore, this work presents the design of an optimized QoS-based packet scheduling architecture to achieve a graceful tradeoff between priority and fairness to all traffics as a solution to the transmission impairments in the evolving growing VoIP networks. The work addresses the impact of delay (latency) and packet loss on the perceived voice quality in constrained bandwidth VoIP networks. The proposal models a structured approach of adaptively evaluating and policing incoming IP flows as well as classifying and mapping different traffic types for individual applications or users. To implement this architecture, an Internet Service Provider's (ISP's) network shall be configured accordingly.

\section{EARLIER PROPOSALS}

Earlier works in optimizing the QoS of a VoIP network have been focused on traffic-scheduling algorithms to ensure either minimum traffic delay constraints or fair resource sharing to all applications running on the network. A QoS-guaranteed network normally differentiates between different types of traffic and provides different treatments to the traffics. This is made possible by using either the type-of-service (TOS) [13] bits or the differentiated services (DiffServ) [14, 15, 16, 17, $18,19,20]$ field in the IP header, or still through the use of signalling protocols such as: resource reservation protocol (RSVP) [21, 22] and multi-protocol label switching (MPLS) [23]. Traffic identification can also be implemented by configuring network devices to support prioritization based on physical port, protocol, IP address, transport address or packet length [8]. In the quest to optimize the QoS of VoIP networks, many schemes have been proposed to address the demands of both delay-sensitive and best effort traffics [12, 24, 25, 26, 27 , $28,29]$. Some approaches aim at preventing congestion by limiting load and using priority scheduling. In all, low latency, jitter and packet loss to the streaming flows are guaranteed only if the rate of the streaming flow is relatively a very small fraction of the link rate $[12,30]$.

\section{DESIGN OF AN OPTIMIZED QoS SCHEDULER FOR VOIP NETWORKS}

Coming from the background that no single technology and model can effectively solve the quality of service (QoS) issues of the evolving growing VoIP system [28], a hybrid scheme is hereby designed. It should be recalled that voice traffic and business/mission critical data (B/MCD) traffic are delay and jitter sensitive. A structured design approach was adopted considering the following design tools: simplicity, modularity, well-formed design, robustness and consistency. The incoming source traffic is generated at the sender side of the network. It is sent to the destination through QoS configured network devices (switches and routers) at the edge of the network.

The aim of the proposed scheduler is to reduce the variable delays and packet loss encountered by traffics (especially voice and business/mission critical data) so as to enhance the quality of service offered by a VoIP Network for the achievement of the inherent benefits of the emerging converged Information and Communication Technology (ICT) industry. The objectives are as follows:

(a) To meet the demands of the expected increase in the volume of voice as well as business/mission critical data (B/MCD) traffics' delay constraints by assigning their packets with the highest priority;

(b) To ensure fair resource sharing among all traffics (real-time and non real-time) in the network;

(c) To adaptively maintain good (optimized) voice quality in a constrained bandwidth IP network without over provisioning the users, thereby achieving a graceful tradeoff between priority and fairness to all traffics.

\subsection{Overview of Scheduler Architecture}

The proposed optimized QoS architecture is an integration of several technologies. It is comprised of the Packet Classifier, the Token Bucket, the Differentiated Services (DiffServ) and the Weighted Round Robin (WRR) Scheduler modules [31, $32,12,29]$. The hybrid architecture is illustrated in Figure 1.

The Packet Classifier module consists of two packet classifiers. Classifier1 is used to classify the packets of the incoming source traffics (p) into two main classes, namely: voice (p1) and non-voice (p2) flows. Packet Classifier2 is used to classify the non-voice flows into two other classes, namely: business/mission-critical data (B/MCD, p3) and others ( $\mathrm{p} 4$ - consisting of video and remaining data traffics). The essence of Classifier2 (which accounts for a major contribution of this work) is to capture and accord business/mission-critical data flows the necessary priority and fairness they deserve.

The dynamics of network traffic flow and packet distinguishing is implemented by the input service routine architecture (packet admission control mechanism), which applies traffic congestion avoidance controls [33, 34] to the incoming flows and places incoming packets into separate queues for subsequent processing by inspecting the type-ofservice (TOS) [13] bits in the packet IP header. Standard firstin-first-out (FIFO) [35] scheduling is employed for forwarding the outputs of classifiers 1 and 2 to the Token Bucket.

The Token Bucket module is used to split the incoming voice or business/mission-critical data (B/MCD) traffic into two sub-flows. The first sub-flow is a well shaped flow with maximum rate equal to $\gamma$ bits/second generated by the Token Bucket. The second sub-flow is the packet ( $\mathrm{p} 5$ - still of voice or $\mathrm{B} / \mathrm{MCD}$ traffic) rejected by the Token Bucket.

In the DiffServ module, video traffic is mapped to Assured Forwarding (AF) [36] traffic class. Voice or $\mathrm{B} / \mathrm{MCD}$ traffic, which was rejected from the Token Bucket is mapped to the Expedited Forwarding (EF) [37] traffic class. The remaining data traffic (such as email, file transfer, and so on) is mapped by default to the Best Effort (BE) class.

The WRR scheduler module is used to adaptively regulate the bandwidth utilization among the competitive traffic flows from the DiffServ module. The output bandwidth is divided into two parts, namely: the reserved (dedicated) link and the shared link. The reserved link is used to service the specified portion of voice or $\mathrm{B} / \mathrm{MCD}$ traffic from the Token Bucket. The shared link is used to service the other traffics as scheduled fairly and adaptively by the WRR scheduler. 


\subsection{An Algorithmic Analysis of the Proposed Scheduler}

The pseudo code of the developed scheduling algorithm is presented in Table 1. Using the top-down design approach, the following algorithm defining the various activities performed at every level of abstraction (module) is analyzed:

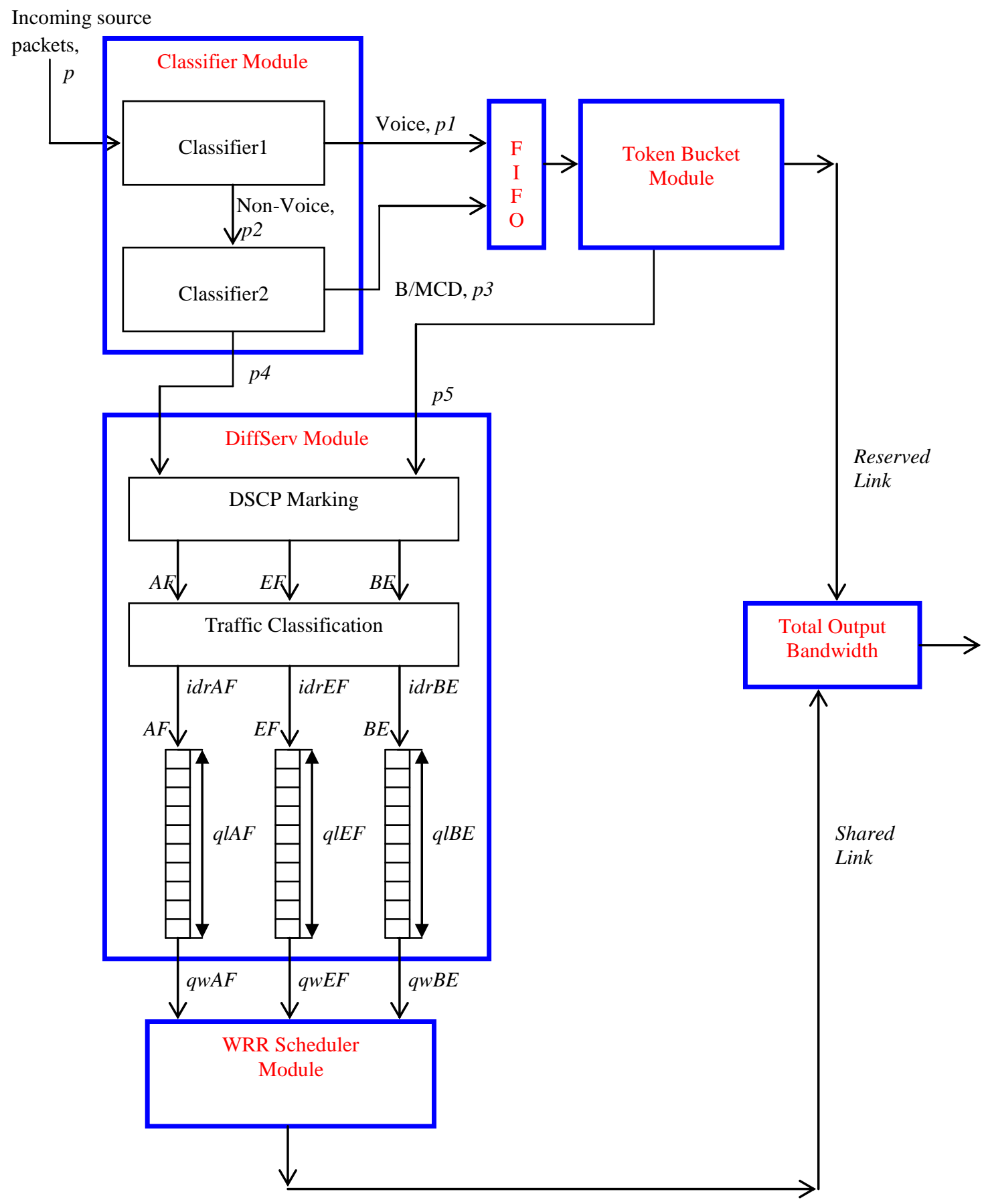

Legend

$i d r A F$, idrEF and $i d r B E=$ average input data rate of $\mathrm{AF}, \mathrm{EF}$ and $\mathrm{BE}$ traffics.

$q l A F, q l E F$ and $q l B E=$ average queue length for $\mathrm{AF}, \mathrm{EF}$ and $\mathrm{BE}$ traffics.

$q w A F, q w E F$ and $q w B E=$ queue weight or service rate for $\mathrm{AF}, \mathrm{EF}$ and $\mathrm{BE}$ traffics.

Figure 1: The optimized hybrid scheduler architecture 
Table 1: Scheduling Control Algorithm of the Optimized Model

\begin{tabular}{|c|}
\hline QoS \\
\hline Packet Classifier \\
\hline $\begin{array}{l}\text { 1. On the arrival of IP packet }(p) \text { to the scheduler } \\
\text { 2. Class = Classifier1 }(p 1) \text { : } \\
\text { 3. If (class = voice }(p 1)) \text { then } \\
\text { 4. } \quad \text { Send to the Token Bucket; } \\
\text { 5. Else } \\
\text { 6. Send to (Classifier } 2(p 2)) \text {; } \\
\text { 7. If (class = business/mission critical data }(p 3)) \text { then } \\
\text { 8. } \quad \text { Send to the Token Bucket; } \\
\text { 9. Else } \\
\text { 10. Send to (DiffServ }(p 4)) ;\end{array}$ \\
\hline Token Bucket \\
\hline $\begin{array}{l}\text { 11. On arrival of IP packets }(p 1),(p 3) \text { to the Token } \\
\text { Bucket } \\
\text { 12. If }(\text { size }(p 1) \text { or }(p 3))<=\text { current token) then } \\
\text { 13. Send to the reserved link; } \\
\text { 14. Else } \\
\text { 15. Send to (DiffServ }(p 5)) \text {; }\end{array}$ \\
\hline DiffServ \\
\hline $\begin{array}{l}\text { 16. On arrival of IP packet }(p 4) \text { to the DiffServ } \\
\text { module } \\
\text { 17. If (traffic = video) then } \\
\text { 18. Mark with AF DSCP value; then } \\
\text { 19. Classify as AF traffic; then } \\
\text { 20. Assign logical queue to traffic; then } \\
\text { 21. Calculate queue length: } q l_{\mathrm{AF}} \text {; then } \\
\text { 22. Send to (WRR scheduler); } \\
\text { 23. Else } \\
\text { 24. Mark by default as BE DSCP value; then } \\
\text { 25. Classify as BE traffic; then } \\
\text { 26. Assign logical queue to traffic; then } \\
\text { 27. Calculate queue length: } q l_{\mathrm{BE}} \text {; then } \\
\text { 28. Send to (WRR scheduler); } \\
\text { 29. On arrival of IP packet ( } p 5) \text { to the DiffServ } \\
\text { module } \\
\text { 30. Mark with EF DSCP value; then } \\
\text { 31. Classify as EF traffic; then } \\
\text { 32. Assign logical queue to traffic; then } \\
\text { 33. Calculate queue length } q l_{\mathrm{EF}} \text {, then } \\
\text { 34. Send to (WRR scheduler); }\end{array}$ \\
\hline WRR Scheduler \\
\hline $\begin{array}{l}\text { 35. Observe the current queue lengths: } q l_{\mathrm{AF}}, q l_{\mathrm{BE}} \text { and } \\
q l_{\mathrm{EF}} \text {; } \\
\text { 36. Observe traffic priority: } \mathrm{P}_{\mathrm{AF}}=2, \mathrm{P}_{\mathrm{BE}}=1 \text { and } \mathrm{P}_{\mathrm{EF}}=3 \text {; } \\
\text { 37. Compute queue (service) weight: } q w_{\mathrm{AF}}, q w_{\mathrm{BE}} \text { and } \\
q w_{\mathrm{EF}} ; \\
\text { 38. If } \mathrm{Max}_{\mathrm{QW}}=\text { maximum of }\left(q w_{\mathrm{AF}}, q w_{\mathrm{BE}}, q w_{\mathrm{EF}}\right) \text {; then } \\
\text { 39. Send to shared link; } \\
\text { 40. Else } \\
\text { 41. If } q w_{\mathrm{AF}}=q w_{\mathrm{BE}}=q w_{\mathrm{EF}} \text {; then } \\
\text { 42. Send to shared link using priority value; } \\
\text { 43. Return Max } \mathrm{O}_{\mathrm{QW}} \text {. }\end{array}$ \\
\hline
\end{tabular}

The Packet Classifier Module: The incoming source packet, $p$ is classified (by Classifier1) as it arrives the edge network device into two classes, namely: voice, $p 1$ and non-voice, $p 2$ (lines 1-2). If the packet is voice, it is directed to the Token
Bucket module (lines 3-4), otherwise it is directed to packet Classifer2 (lines 5-6). If the non-voice packet is business/mission critical data (B/MCD), it is directed also to the Token Bucket module (lines7-8), otherwise it is directed to the DiffServ module (lines 9-10).

The Token Bucket Module: The voice or B/MCD flow directed to the Token Bucket module is divided into two parts. The first part (the reserved voice or B/MCD flow) is well shaped with a maximum rate equal to $\gamma$ bits/second (lines 1112 ). The second part comprises the voice or $\mathrm{B} / \mathrm{MCD}$ flow that is rejected from the Token Bucket module because of overflowing the rate $\gamma$ (line 14). The reserved voice or $\mathrm{B} / \mathrm{MCD}$ flow is served directly through a reserved link dedicated only for this purpose (line 13).

The DiffServ Module: The surplus voice or B/MCD flow (rejected from the Token Bucket) is redirected to the DiffServ module (line 15) where it is marked and classified as EF traffic (lines 29-31). Also, if the non-voice flow ( $p 4)$ from Classifier2 is video, it is marked and classified as AF traffic (lines 16-19), otherwise it is marked and classified by default as BE traffic (lines 23-25). The three classes of traffic are respectively assigned logical queues, the length of each queue is calculated and the queues directed to the WRR scheduler module (lines 32-34, 20-22 and 26-28).

The WRR Scheduler Module: The current queue length and priority of each of the traffic classes are observed (lines 3536). The observed parameters are used to compute the queue (or service) weight of each of the traffic classes (line 37). The traffic class that has the maximum weight is serviced first, then the next, and so on (lines 38-39). If the computed weights are equal to each other, the traffic class that has the highest priority value is serviced first, then the next, and so on for every round-robin session (lines 40-42). This process is repeated for as long as there are available traffic queues for transmission (line 43).

Please note that the average input data rates ( $(i d r A F$, $i d r E F$ and $i d r B E)$ ) of $\mathrm{AF}, \mathrm{EF}$ and $\mathrm{BE}$ traffics are respectively used to compute the average queue lengths ( $q l A F$, qlEF and $q l B E)$ for $\mathrm{AF}, \mathrm{EF}$ and $\mathrm{BE}$ traffics. The average queue lengths are in turn used to compute the queue weight or service rate $(q w A F, q w E F$ and $q w B E)$ for $\mathrm{AF}, \mathrm{EF}$ and $\mathrm{BE}$ traffics.

\subsection{Signal Flowchart of the Optimized Architecture}

The product of integrating all the functional modular pieces is a typical signal flowchart, which is given in Figure 2.

\section{EVALUATION AND DISCUSSION}

The designed hybrid scheduler architecture of Figure 1 and algorithm of Table 1 incorporate mechanisms for: accommodating the demands of the expected rapid increase in the volume of voice traffic as PSTN progressively migrates to VoIP; according precedence to the delay-sensitive voice and business/mission critical data $(\mathrm{B} / \mathrm{MCD})$ traffic flows to reduce the transmission impairment factors; ensuring fair resource sharing among all traffic flows (real-time and non real-time) in the network, and adaptively maintaining optimal voice quality without over provisioning the users.

The design has been structured to be simple and easy to understand. It is developed in a modular form for easy manipulation. The structure also makes the proposed architecture robust and consistent in its operation. The algorithmic analysis explains the functional sequence for the optimal performance of the designed architecture. The signal flowchart of Figure 2 presents a clearer view of the design and 
analysis of the functionality of the optimized QoS-based packet scheduler. The inbuilt functionalities ensure that the demands of the expected rapid increase in the volume of voice traffic are adequately met as the traditional telephone services progressively migrate into the emerging converged VoIP network.

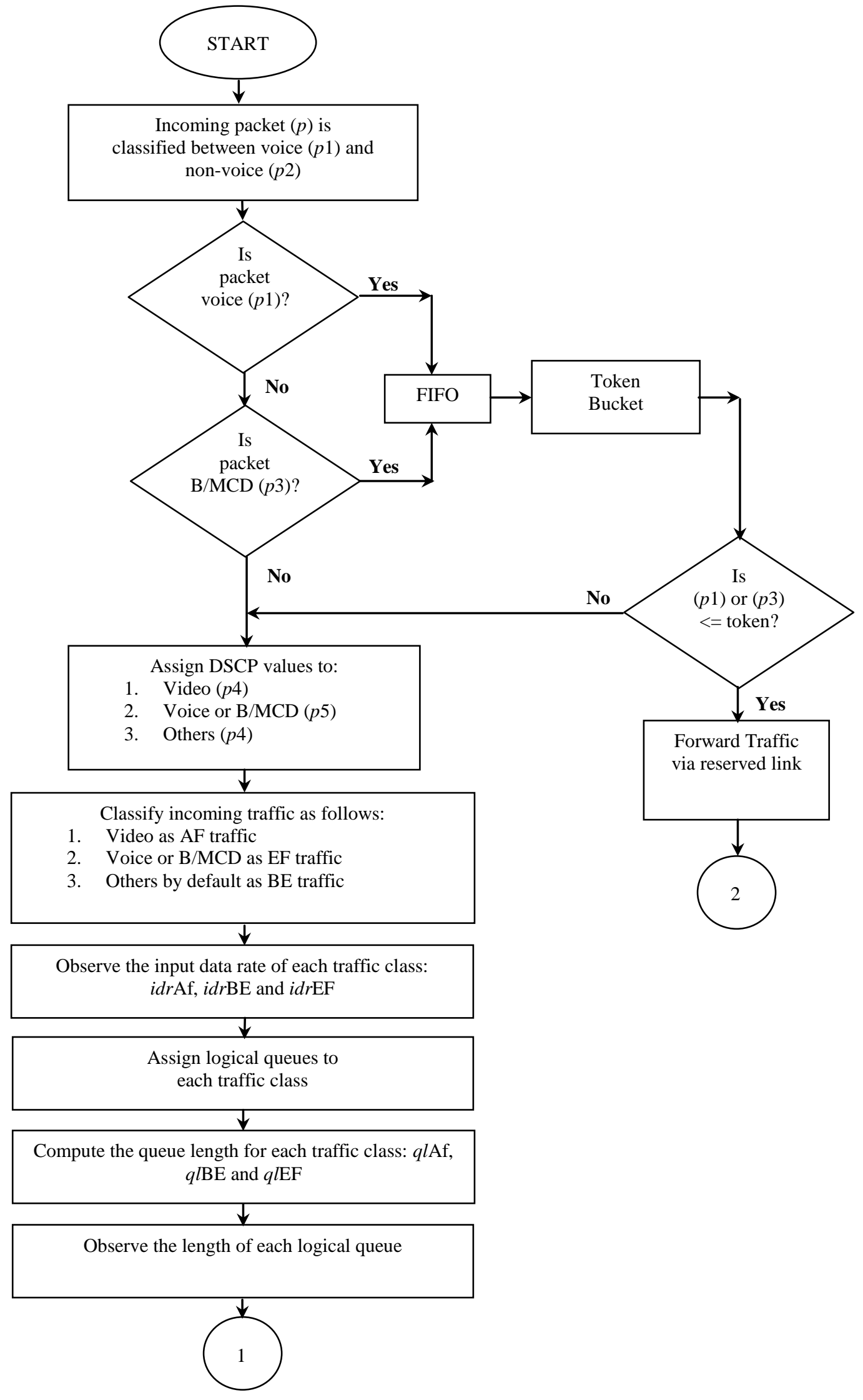

Figure 2: Structured Flowchart of the QoS-based Packet Scheduler 


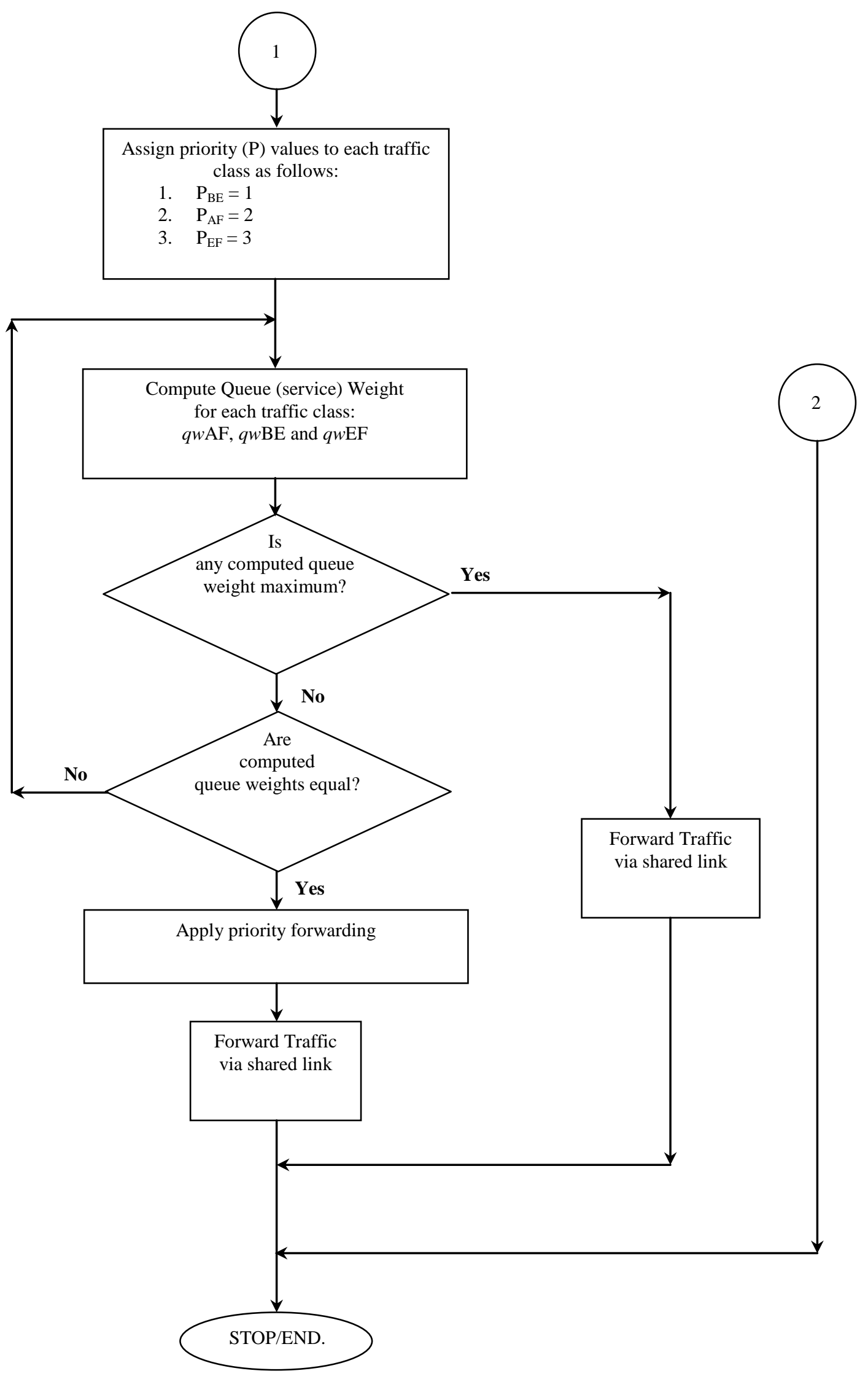




\section{CONCLUSIONS}

In this work, the design of a hybrid packet scheduler architecture, scheduling control algorithm and structured signal flowchart as well as the algorithmic analysis of an efficient packet scheduler for optimizing the QoS of VoIP networks have been carried out. Adequate mechanisms have been incorporated to capture the demands of business/mission critical data $(\mathrm{B} / \mathrm{MCD})$ and the expected rapid increase in the volume of voice traffic in the emerging VoIP network. Earlier proposals to the QoS challenges of VoIP networks have considered $\mathrm{B} / \mathrm{MCD}$ traffics as best-effort flow. The design also addressed the problems of network resource sharing and transmission impairment factors. The result is an efficient packet scheduling architecture for optimizing the QoS of VoIP networks that achieves and ensures a graceful tradeoff between priority and fairness to all traffic flows running on the network.

Well-formed and reliable QoS schemes are indeed very expensive. But this work offers a great alternative in economy and reliability. It provides the necessary confidence that network designers, operators and administrators need to effectively monitor and manage real-time and business/mission critical data as well as other best effort traffic flows. The work has also opened a doorway for more academic/research work in the emerging technologies of the converged ICT industry.

\section{ACKNOWLEDGMENTS}

This work is supported by the MacArthur Foundation Sub Grant, University of Port Harcourt.

\section{REFERENCES}

[1] Ash, G. R., 2007. Trafic Engineering and QoS Optimization of Integrated Voice and Data Networks. Elsevier Inc.

[2] Mousavipour, F. and Khosravipour, M. J., 2012. VoIP Quality Enhancement with Wideband Extention Method in Broadband Networks. IEEE Latin America Transactions, Vol. 10, No. 1, pp 1190-1194.

[3] Perlicki, K., 2002. Simple Analysis of the Impact of Packet Loss and Delay on Voice Transmission Quality, Journal of Telecommunication and Information Technology (JTIT), February, pp 53-56; [Online], Available:

http://www.nit.eu/czasopisma/JTIT/2002/2/53.pdf

(Document accessed: October 27, 2013).

[4] Altera Corporation, 2000. Implementing Voice over Internet Protocol, Application Note 128, Ver.1.1, Altera Corporation, San Jose. [Online]. Available on: http://www.altera.com/search?output=xml_no_dtd\&sort= date $\% 3 \mathrm{AD} \% 3 \mathrm{AL} \% 3 \mathrm{Ad} 1 \& \mathrm{client}=\mathrm{www}$ frontend\&proxy stylesheet $=$ www_frontend \&ie $=U T F-8 \& o e=U T F-$ $8 \&$ site $=w w w \& q=$ Implementing $\% 20$ Voice $\% 20$ over $\% 20 \mathrm{I}$ nternet\%20Protocol (Document accessed: December 8, 2009).

[5] Thomsen, G. and Jani, Y., 2000. 'Internet Telephony: Going Like Crazy, IEEE Spectrum, pp 52-58.

[6] Denisowski, P., 2001. How Does It Sound?, IEEE Spectrum.

[7] Narcisi, G., 2013. VoIP vs PSTN: VoIP heats up as the PSTN moves into Retirement, Search Unified Communications, TechTarget. [Online], (Document accessed: May 8, 2013) Available on: http://searchunifiedcommunications.techtarget.com/news /2240177294/VoIP-vs-PSTN-VoIP-heats-up-as-thePSTN-moves-into-retirement

[8] Ma, A., 2001. Voice over IP (VoIP), VoIP Book, Spirent Communications Inc., SmartBits Performance Analysis System. [Online], (Document accessed on: December 8, 2012). Available on http://www.phonet.cz/archiv/dok_cizi/Spirent_100.pdf

[9] Cisco Systems, 2008. Understanding Delay in Packet Voice Networks, White Papers, Document ID: 5125, Cisco Systems Inc. [Online], Available: http://www.cisco.com/image/gif/paws/5125/delaydetails.pdf (Document accessed: December 12, 2013).

[10] NetPredict, 2004. Assess the ability of your Network to handle VoIP before you Commit, White Paper, NetPredict, Inc. [Online], Available: www.netpredict.com/pdfs_all/WhitePaper-VoIP.pdf (Document accessed: December 8, 2006).

[11] Cisco Systems, 2013. Understanding Jitter in Packet Voice Networks, Cisco IOS Platforms, Document ID: 18902, Cisco Systems Inc. [Online], Available: http://www.cisco.com/image/gif/paws/18902/jitter_packe t_voice.pdf (Document accessed: December 9, 2013).

[12] Ahmed, S., Jiang, X. and Horiguchi, S., 2007. Efficient Scheduler for the Growing VoIP Traffic, International Conference on Parallel Processing Workshops (ICPPW), IEEE Computer Society.

[13] Almquist, P., 1992. Type of service in the internet protocol suite, Request for Comments (RFC) (Proposed Standard) 1349, Internet Engineering Task Force (IETF).

[14] Blake, S., Black, D., Carlson, M., Davies, E., Wang, Z. and Weiss, W., 1998. An Architecture for Differentiated Services, IETF RFC 2475.

[15] Clark, D. D. and Wroclaski, J., 1997. An Approach to Service Allocation in the Internet, IETF Internet Draft [online] Available: http://tools.ietf.org/pdf/draft-clarkdiff-svc-alloc-00.pdf, (Document accessed: July 14, 2013).

[16] Nichols, K., Blake, S., Baker, F. and Black, D., 1998 Definition of the differentiated services field (DS field) in the IPv4 and IPv6 headers, Request for Comments (RFC) (Proposed Standard) 2474, Internet Engineering Task Force.

[17] Nichols, K., Jacobson, V. and Zhang, L., 1999. A two-bit Differentiated Services Architecture for the Internet, Network Working Group RFC 2638, Internet Engineering Task Force.

[18] Angulo-Bernal, M., Turrublartes-Reynaga, M. A., Torres-Roman, D. L. and Argumedo-Ledon, M. A., 2004. Voice and Data Applications in Differentiated Service Intranets, Journal of Applied Research and Technology, Vol. 2, No. 1, pp 62-67. [Online], (Document viewed: 08/12/13) Available on: http://www.redalyc.org/pdf/474/47420106.pdf

[19] Cisco Systems, 2008. Quality of Service - The Differentiated Services Model, Cisco Public Information, Cisco Systems Inc. [Online], (Document accessed: July 14, 2013). Available: http://www.cisco.com/en/US/prod/collateral/iosswrel/ps6 
537/ps6558/ps6610/product_data_sheet0900aecd8031b3 6d.html.

[20] Cisco Systems, 2008. Implementing Quality of Service Policies with DSCP, Document ID: 10103, Cisco Systems Inc. [online], Available: http://www.cisco.com/en/US/tech/tk543/tk757/technolog ies_tech_note09186a00800949f2.shtml and http://www.cisco.com/image/gif/paws/10103/dscpvalues. pdf (Document viewed: September 28, 2013).

[21] Braden, R., Zhang, L., Berson, S., Herzog, S. and Jamin S., 1997. Resource Reservation Protocol (RSVP), Network Working Group RFC 2205, Internet Engineering Task Force.

[22] Cisco Systems, Resource Reservation Protocol (RSVP), Cisco Systems Inc. [Online], Available: http://www.cisco.com/en/US/products/ps6652/products_i os_protocol_option_home.html (Document accessed: July 14, 2013).

[23] Rosen, E., Viswanathan, A. and Callon, R., 2001. Multiprotocol Label Switching Architecture, Network Working Group RFC 3031, Internet Engineering Task Force.

[24] Gardner, M. T., Frost, V. S. and Petr, D. W., 2003. Using Optimization to Achieve Efficient Quality of Service in VoIP Networks, Proceedings of the IEEE International Conference on Performance, Computing and Communications, pp 475-480.

[25] Bandung, Y., Machbub, C., Langi, A.Z.R. and Supangkat, S.H., 2008. Optimizing Voice ocer Internet Protocol (VoIP) Networks based on Extended E-Model, IEEE Conference on Cybernetics and Intelligent Systems, Chengdu, pp 801-805.

[26] Fischer, M. J., Masi, D. M. B. and Shortle, J. F., 2008. Approximating Low Latency Queuing Buffer Latency, Fourth Advanced International Conference on Telecommunications, IEEE Computer Society, pp 188194.

[27] Culverhouse, M. E., Ghita, B. V., Reynolds, P. and Wang, X., 2010. Optimising Quality of Servise through the Controlled Aggregation of Traffic, International Conference for Internet Technology and Secured Transactions (ICITST), London.
[28] Tu, C., 2011. Study on QoS Protection Mechanism of VoIP Systems, International Symposium on Intelligence Information Processing and Trusted Computing, IEEE Computer Society, pp 151-153.

[29] Chaudhuri, S. G., Kumar, C. S. and RajaKumar, R. V., 2012. Validation of a DiffServ based QoS Model Implementation for Real-Time Traffic in a Test Bed, IEEE National Conference on Communications (NCC).

[30] Pitts, J. M. and Schormans, J. A., 2006. Configuring IP QoS Mechanisms for Graceful Degradation of Real-Time Services, IEEE Military Communications Conference (MILCOM), Washington DC, pp 1-7.

[31] Chimento, P. F., 2000. Standard Token Terminology, Unpublished working document. [Online], (Document accessed: November 8, 2013). Available: http://qbone.internet2.edu/bb/Traffic.pdf

[32] Zhao, W., Olshefski, D. and Schulzrinne, H., Internet Quality of Service: An Overview, Columbia University Research Report CUCS-003-00, IBM Research, [Online], (Document accessed on July 3, 2013), Available: http://www.research.ibm.com/people/o/olshef/publicatio ns/cucs-003-00.pdf

[33] Demers, A., Keshav, S. and Shenker, S., 1989. Design and Analysis of a Fair Queuing Algorithm, Proceedings of ACM SIGCOMM, Austin, pp 1-12.

[34] Golestani, S. J., 1994. A Self-Clocked Fair Queueing Scheme for Broadband Applications, In Proceedings of IEEE INFOCOMM, pp 636-646.

[35] Kurose, J. F. and Ross, K. W., 2000. Scheduling and Policing Mechanisms, Scheduling and Policing Mechanisms for Providing QoS Guarantees. [Online], (Document accessed: November 8, 2013). Available: http://jpkc.ncwu.edu.cn/jsjwl/net/ross/book/emerge/sched uling_and_policing.htm

[36] Heinanen, J., Baker, F., Weiss, W. and Wroclawski, J., 1999. Assured Forwarding PHB Group, vol. IETF RFC 2597.

[37] Davie, B., Charny, A., Bennet, J. C. R., Benson, K., LeBoudec, J. Y., Courtney, W., Davaei, S., Firoiu, V. and Stiliadis, D., 2002. An Expedited Forwarding Perhop Behaviour (PHB), IETF RFC 3246. 